\title{
Robust Watermarking through Dual Band IWT and Chinese Remainder Theorem
}

\author{
Prajanto Wahyu Adi ${ }^{1}$, Farah Zakiyah Ramhanti ${ }^{2}$, Edy Winarno ${ }^{3}$ \\ ${ }^{1,2}$ Department of Informatics Engineering, Universitas Dian Nuswantoro, Semarang, Indonesia \\ ${ }^{3}$ Faculty of Information Technology, Universitas Stikunank, Semarang, Indonesia
}

\section{Article Info \\ Article history: \\ Received Sep 06, 2017 \\ Revised May 20, 2018 \\ Accepted Oct 10, 2018}

\section{Keywords:}

Chinese Remainder Theorem IWT

Watermarking

\begin{abstract}
CRT was a widely used algorithm in the development of watermarking methods. The algorithm produced good image quality but it had low robustness against compression and filtering. This paper proposed a new watermarking scheme through dual band IWT to improve the robustness and preserving the image quality. The high frequency sub band was used to index the embedding location on the low frequency sub band. In robustness test, the CRT method resulted average NC value of $0.7129,0.4846$, and 0.6768 while the proposed method had higher NC value of $0.7902,0.7473$, and 0.8163 in corresponding Gaussian filter, JPEG, and JPEG2000 compression test. Meanwhile the both CRT and proposed method had similar average SSIM value of 0.9979 and 0.9960 respectively in term of image quality. The result showed that the proposed method was able to improve the robustness and maintaining the image quality.
\end{abstract}

Copyright $@ 2018$ Institute of Advanced Engineering and Science. All rights reserved.

\section{Corresponding Author:}

Prajanto Wahyu Adi,

Departement of Informatics Engineering,

Universitas Dian Nuswantoro (UDINUS),

Jl. Imam Bonjol 207, Semarang 50131, Indonesia.

Email: prajanto@dsn.dinus.ac.id

\section{INTRODUCTION}

The increasing number of digital media violation has increased the concern about the digital media protection. One of the ways to resolve the issue is the watermarking method which becomes rapidly developed in the last decade. Watermarking is a way of protecting the digital media by inserting certain information called watermark into the media [1]. The watermark contains copyright information that can be used as proof of legal ownership. Watermarking methods can be implemented on most of digital media such documents, audio, image, and video. In current development, image becomes the most used popular medium in watermarking due to its capability of keeping the watermark invisible to the human eye especially in heterogeneous area.

Watermarking methods can be classified into spatial and transform domain methods. Spatial domain method is mean of embedding the watermark on the image pixels directly and it has lower complexity but less in robustness. Alternatively, the transform domain methods are embeds the watermark on the transformed pixels called coefficient in various frequency levels. They have better robustness for retaining the watermark [2]. Several development of watermarking in transform domain methods have been conducted such Discrete Cosine Transform (DCT) [3]-[5]. Discrete Wavelet Transform (DWT) [6]-[9], Integer Wavelet Transform (IWT) [10].

DCT is a basic transform method that widely used but it can degrade the image significantly when embedding large amount of watermark. The more advanced method DWT is able to represent an image into visual feature in multiple level of resolution which can produce watermarked image with better imperceptibility and robustness [11]. However, DWT has floating coefficient value that causing reversibility problem. Meanwhile, IWT is the next generation wavelet transformation which can solve the DWT problem 
[12]. It is developed from the DWT using various lifting scheme to reserve the floating point within the range of integer value.

This paper is proposed a new approach to embed the watermark on the integer wavelet domain using Chinese Remainder Theorem (CRT) algorithm. The low and high sub bands of IWT are used in this scheme. The high sub band is used to determine the embedding location on the low sub band and then the watermark bits are embedded using CRT algorithm based on remainders of mutual coprime integer $c_{1}$ and $c_{2}$. The CRT is work on integer range that corresponds with the IWT and has lower requirement in space and complexity which can streamline the process.

\section{RESEARCH METHOD}

The proposed method is aimed to get better robustness within the same imperceptibility compared to the previous CRT method. The Haar IWT is used to decompose host image into four wavelet sub band of LL, HL, LH, and HH. The HH used to maps the embedding location on LL. The watermark bits are then embedded using CRT algorithm with $c_{1}$ and $c_{2}$ value of 6 and 11 respectively. The robustness of the CRT and the proposed methods are measured under Gaussian filter, JPEG, and JPEG2000 compression using standard Normalized Correlation (NC). The next experiment is to measure imperceptibility the both algorithms using Structural Similarity (SSIM) by Wang et al. [13].

\subsection{Haar IWT}

Haar IWT is an efficient integer wavelet that developed from Haar DWT using lifting scheme by Xu et al. [14 ]and many used in recent years by [15]-[17]. It is used simple round down operation to reserve the floating value in the decomposition process as shown in (1) until (4). Haar IWT is decomposed $2 \times 2$ independence blocks of host image below

$$
\left[\begin{array}{cc}
I_{x, y} & I_{x, y+1} \\
I_{x+1, y} & I_{x+1, y+1}
\end{array}\right]
$$

into four sub band as follows:

$$
\begin{aligned}
& L L=\left\lfloor\frac{\left\lfloor\frac{I_{x, y}+I_{x, y+1}}{2}\right\rfloor+\left\lfloor\frac{I_{x+1, y}+I_{x+1, y+1}}{2}\right\rfloor}{2}\right\rfloor \\
& H L=\left\lfloor\frac{I_{x, y}-I_{x, y+1}+I_{x+1, y}-I_{x+1, y+1}}{2}\right\rfloor \\
& L H=\left\lfloor\frac{I_{x, y}+I_{x, y+1}}{2}\right\rfloor-\left\lfloor\frac{I_{x+1, y}+I_{x+1, y+1}}{2}\right\rfloor \\
& H H=I_{x, y}-I_{x, y+1}-I_{x+1, y}+I_{x+1, y+1}
\end{aligned}
$$

where $I_{x, y}$ is an image pixel at row $x$ and column $y$ on each block. This process will convert the host image into four sub band with size of one quarter each. LL is a low frequency sub band that represents average of host image. The next sub bands HL and LH are the middle frequency that contains the horizontal and vertical feature. The last sub band $\mathrm{HH}$ has high frequency coefficient with the diagonal feature in it.

The $\mathrm{HH}$ is used to determine the embedding location based on the absolute value of the coefficients. The highest coefficients value of the watermark bits numbers are then use to embed the watermark on the related position of LL. The reconstruction process is perform to build the watermarked image $\left(I^{\prime}\right)$ from the watermarked block. 


$$
\left[\begin{array}{cc}
I^{\prime}{ }_{m, n} & I^{\prime}{ }_{m, n+1} \\
I^{\prime}{ }_{m+1, n} & I^{\prime}{ }_{m+1, n+1}
\end{array}\right]
$$

using the following equation:

$$
\begin{aligned}
& I_{x, y}^{\prime}=L L+\left\lfloor\frac{L H+1}{2}\right\rfloor+\left\lfloor\frac{H L+\left\lfloor\frac{H H+1}{2}\right\rfloor+1}{2}\right\rfloor \\
& I_{x, y+1}^{\prime}=I_{x, y}^{\prime}-\left(H L+\left\lfloor\frac{H H+1}{2}\right\rfloor\right) \\
& I_{x+1, y}^{\prime}=L L+\left\lfloor\frac{L H+1}{2}\right\rfloor-L H+\left\lfloor\frac{H L+\left\lfloor\frac{H H+1}{2}\right\rfloor-H H+1}{2}\right\rfloor \\
& I_{x, y+1}^{\prime}=I_{x+1, y}^{\prime}-\left(H L+\left\lfloor\frac{H H+1}{2}\right\rfloor-H H\right)
\end{aligned}
$$

In the reconstruction process the Haar IWT is added the integer value of 1 as a lifting scheme as shown in (5) until (8) to restore the rounded float value in the decomposition process. This is ensured perfect reversibility on the reconstruction process of the watermarked image.

\subsection{CRT Algorithm}

CRT is an algorithm that able to reduce the large size calculation within modulo operator to the each of modulus coprime factors [18]. It is able to simplify the determination of integer number from the remainder series using the coprime moduli [19]. Moreover, the CRT can be used as an embedding scheme through the modification of the coprime integers [20]. The 6-bit value of pixel $p$ is defined as follows:

$$
p \bmod c_{i}=r_{i}
$$

where $r_{i}$ is the $i$-th remainder of $p$

$$
p \bmod m=\left(\sum_{i=1}^{s} r_{i} \frac{m}{c_{i}} a_{i}\right)
$$

where $c_{i}$ are coprime integer of $s$ set of the modulus $m$

$$
m=c_{1} \cdot c_{2} \cdot \ldots \cdot c_{s}
$$

from the corresponding $a_{i}$ value

$$
\left(a_{i} \frac{m}{c_{i}}\right) \bmod c_{i}=1
$$

The watermark bit $b$ is embedded on the $p$ through a modification process of 6-bit pixel value $p$ based on the remainders $r_{1}$ and $r_{2}$ from the coprime integers $c_{1}$ and $c_{2}$ as shown in (9). The coprime integers are the mutual coprime values that use to generate the modulus $m$ as shown in (10) until (12). The corresponding value $a$ is determined such that the value of $p$ is fulfilled. The modulus should be larger and closest to the maximum 6-bit value of 63 from the possible coprime integers. 


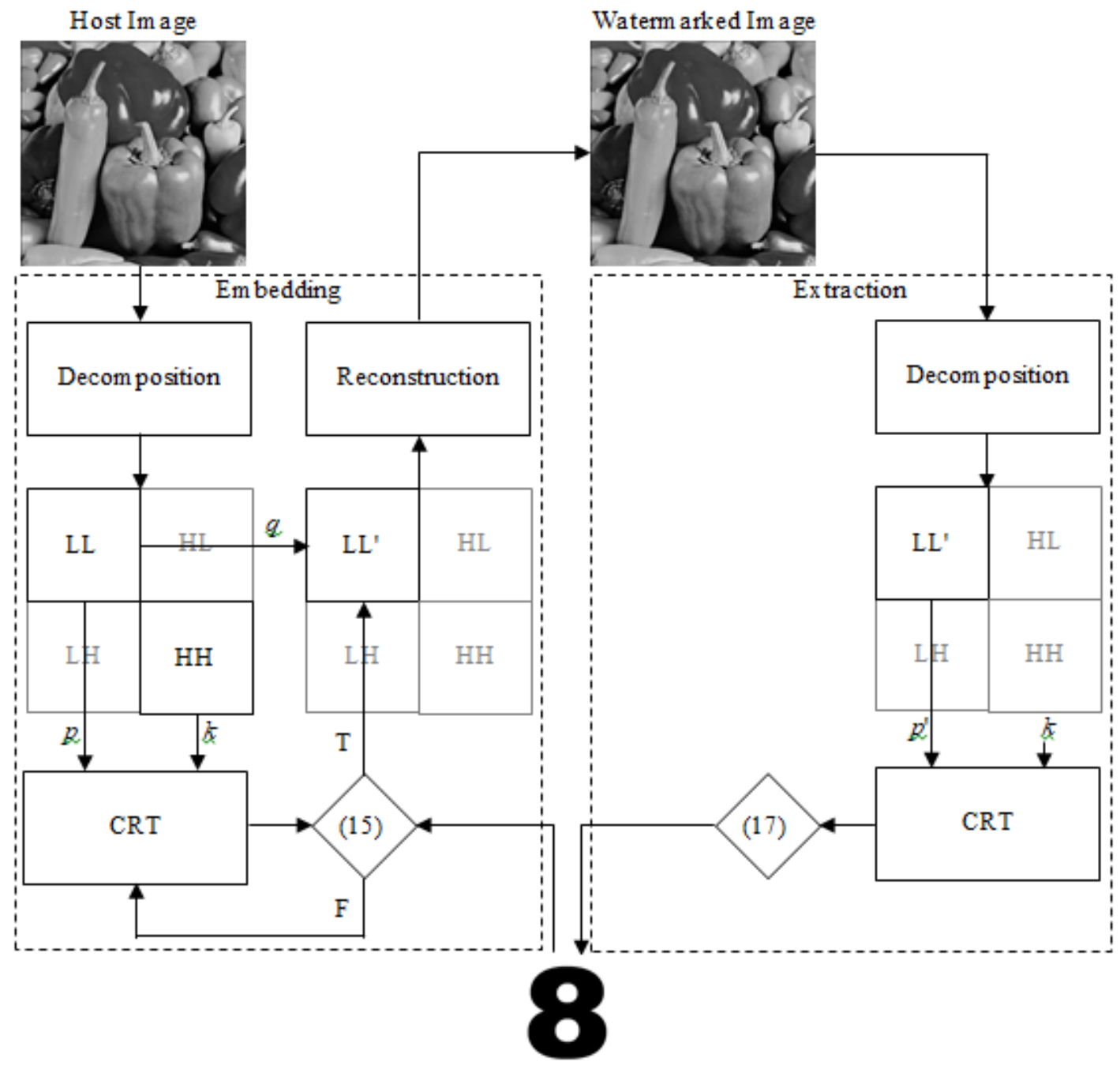

Waterm ark Im age

Figure 1. Proposed method

\subsection{Embedding}

The $p$ value is modified by the addition and subtraction of an incremental value and then the remainders are compared. It is done iteratively until they are fulfilled the condition of watermark bits. The detail embedding process is showed in Figure 1 as follows:

a. Decompose the host image using (1) until (4) into wavelet sub band $L L, H L, L H$, and $H H$

b. Read the number of watermark bits $w$

c. Get the absolute coefficient value of $\mathrm{HH}$ and use the w-most significant value as pixel index $\mathrm{k}$

d. Store the 6-bit integer value as $\mathrm{p}$ and the 2 most significant bits value as $\mathrm{q}$ from the indexed pixels

e. Calculate the remainders $\mathrm{r} 1$ and $\mathrm{r} 2$ of indexed pixel using CRT:

$$
\begin{aligned}
& r_{1}=p \bmod c_{1} \\
& r_{2}=p \bmod c_{2}
\end{aligned}
$$

f. Embed the watermark bit $b$ according into $p_{k}$ based on this condition:

$$
r_{1} \begin{cases}\geq r_{2} & , b=1 \\ <r_{2} & , b=0\end{cases}
$$


g. Add the $q$ into $p$ to get the embedded sub band $L L^{\prime}$

$$
L L^{\prime}=p \oplus q
$$

h. Reconstruct the $L L^{\prime}, H L, L H$, and $H H$ to get the watermarked image.

\subsection{Extraction}

The $k$ value is used for indexing the embedding location on the embedded sub band $L L^{\prime}$ in detail as follows:

a. Decompose the watermarked image using (5) until (8) into wavelet sub band $L L^{\prime}, H L, L H$, and $H H$

b. Get the embedded value $\mathrm{p}^{\prime}$ using $\mathrm{k}$ from the indexed pixel of LL'

c. Calculate the remainders $\mathrm{r} 1$ and $\mathrm{r} 2$ of embedded value using (13) and (14)

d. Determine the bit of watermark using the following condition:

$$
b= \begin{cases}1 & , r_{1} \geq r_{2} \\ 0 & , r_{1}<r_{2}\end{cases}
$$

\section{RESULT AND ANALYSIS}

In this paper, six standard images within size of $512 \times 512$ pixel in grayscale format and a binary image within size of $128 \times 128$ pixel are used as host images and watermarked image respectively as shows in Figure 2.



(a)



(d)

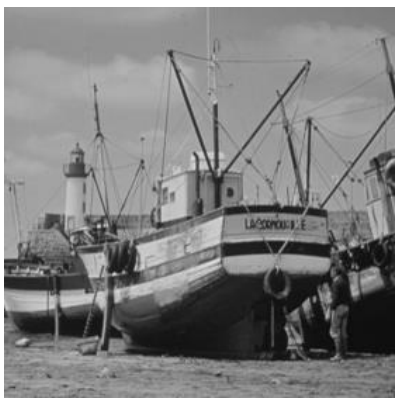

(b)



(e)

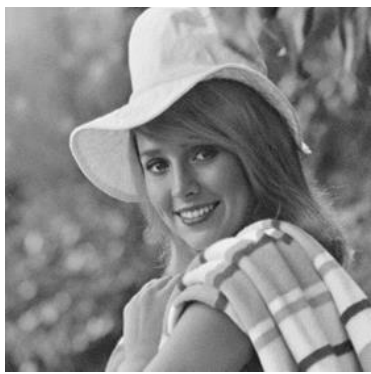

(c)

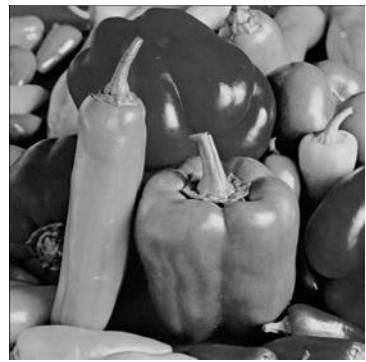

(f)



(g)

Figure 2. The Host Images of (a)Baboon, (b)Boat, (c)Elaine, (d)House, (e)Jet, (f) Peppers, and Watermarked Image $(\mathrm{g})$ 


\subsection{Robustness}

In this section, the robustness of the algorithms are measured under Gaussian filtering, JPEG, and JPEG2000 compressions with the standard sigma, quality factor, and compression ratio of 0.4 , 90, and 3 respectively. The result shows that the proposed method has outperformed the CRT in filtering and compressions test as presented in Table 1, Figure 3, 4, and 5. The CRT method result has the average NC value of $0.7129,0.4846$, and 0.6768 while the proposed method has higher NC value of $0.7902,0.7473$, and 0.8163 in corresponding Gaussian filter, JPEG, and JPEG2000 compression test. This is due to the use of LL that has higher robustness than the direct embedding of CRT. It able to distribute the changes of Gaussian filter and both compression attacks to the down sampled of adjacent pixels evenly. The load partition process has successfully improved the robustness.

Table 1. Robustness

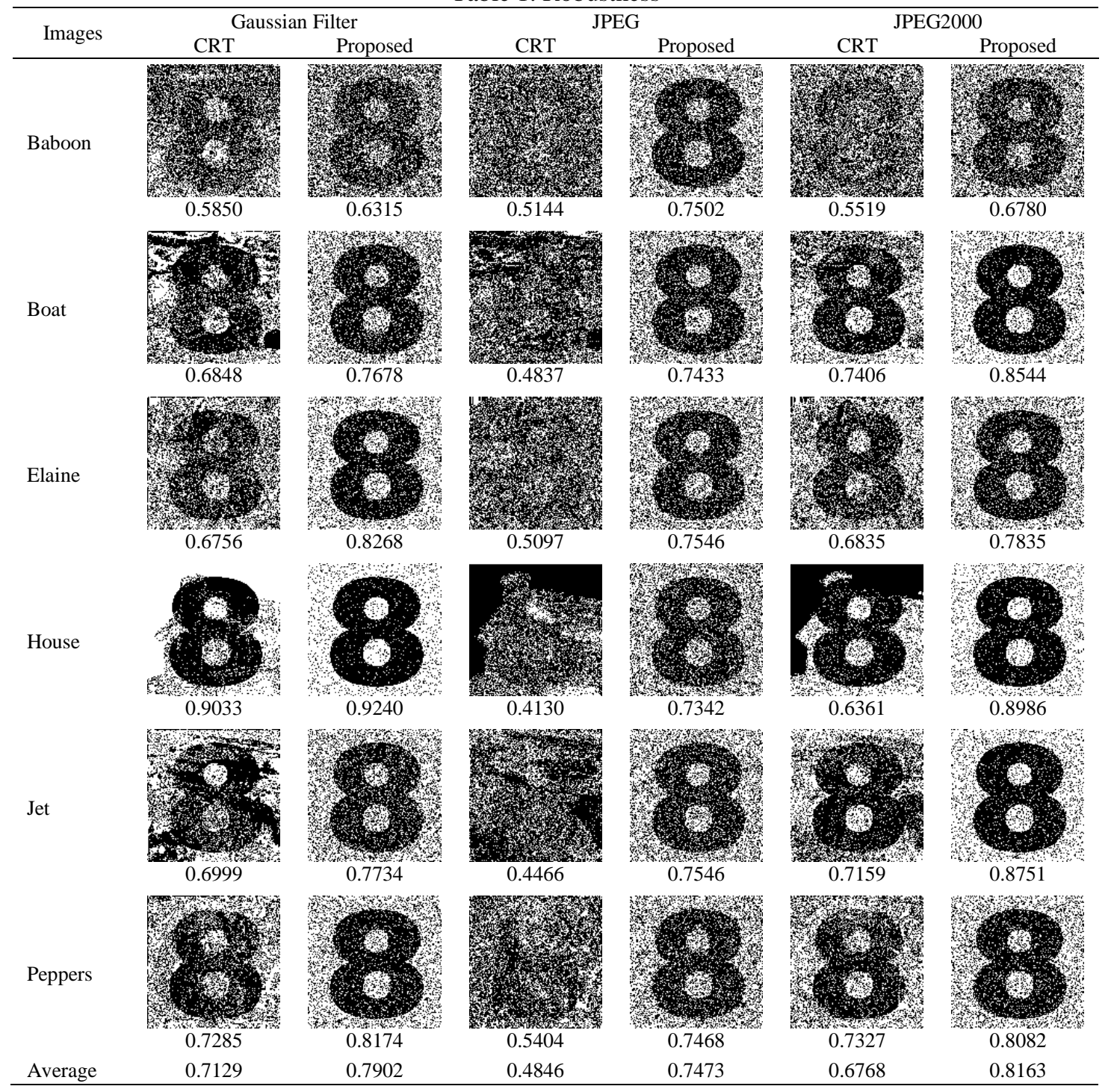




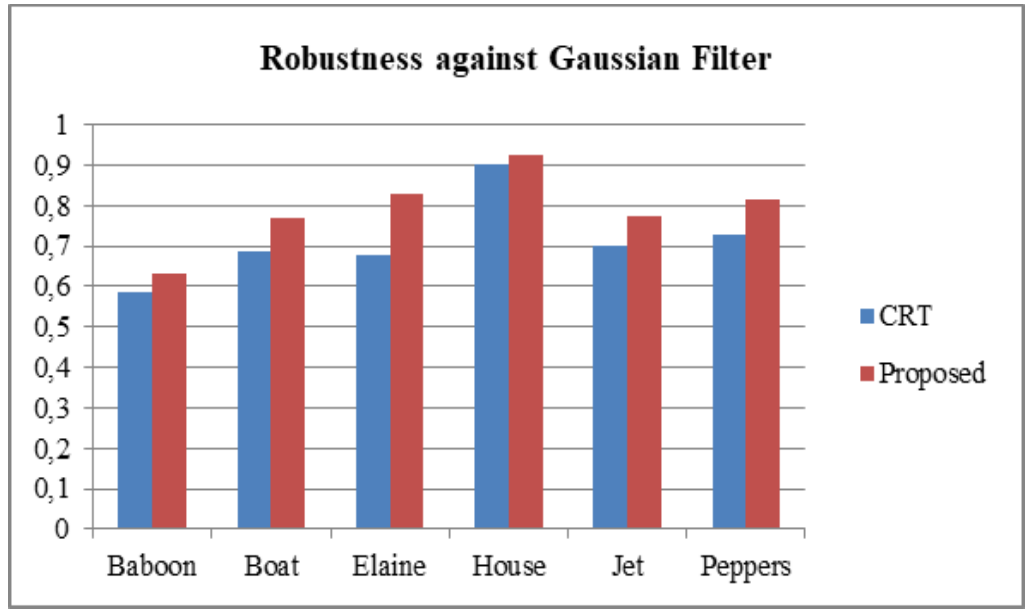

Figure 3. Robustness against Gaussian filter

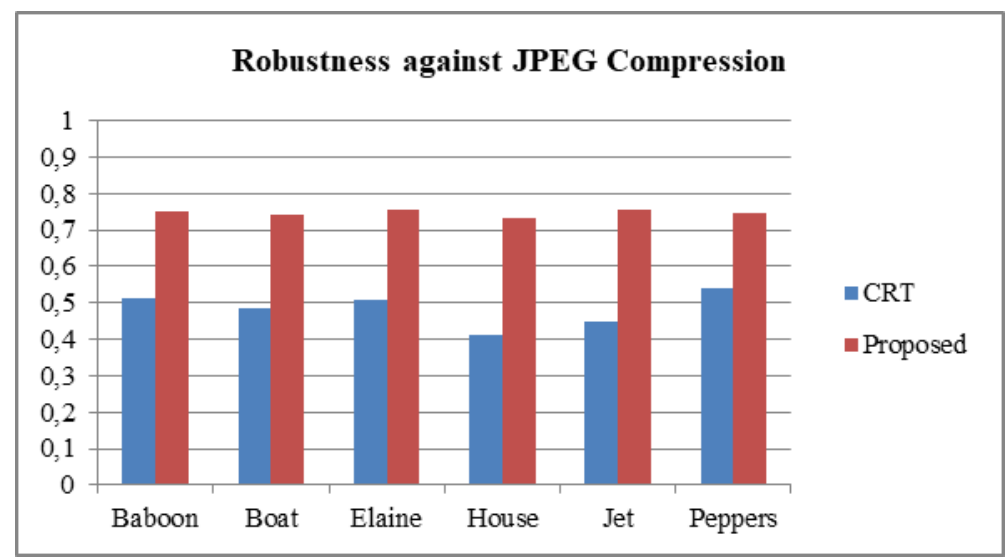

Figure 4. Robustness against JPEG compression

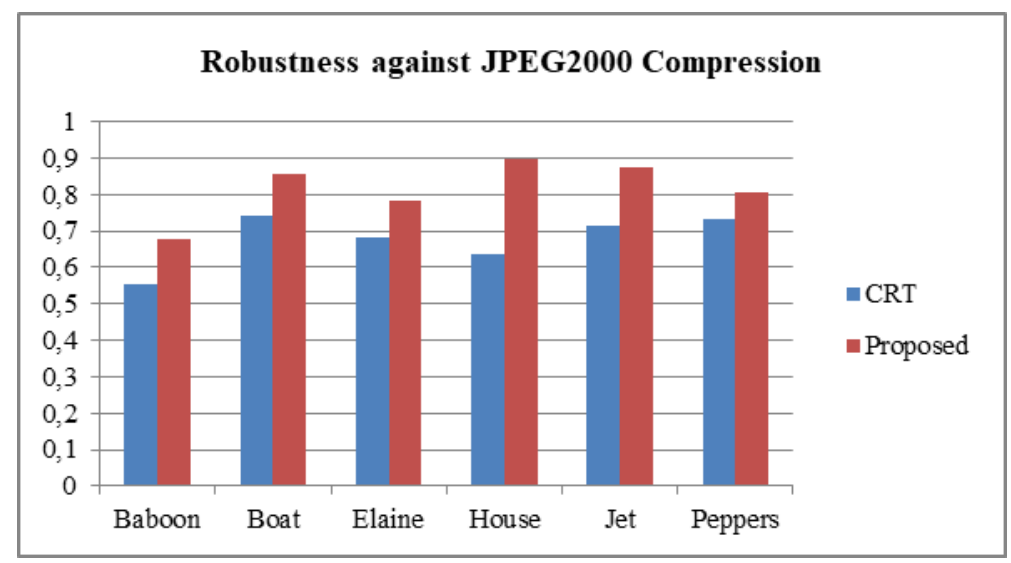

Figure 5. Robustness against JPEG2000 compression

\subsection{Image Quality}

The next experiment is conducted to measure the images quality using standard SSIM due to the accordance with the human visual system. The Table 2 and Figure 6 show that the both algorithms method has similar performance in image quality measurement. The CRT and proposed method have average SSIM 
value of 0.9979 and 0.9960 respectively. The proposed method is used the HH that contains the most significant coefficients which is directly proportional to the pixel frequency.

Table 2. Images Quality

\begin{tabular}{ccc}
\hline Image & CRT & Proposed \\
\hline Baboon & 0.9994 & 0.9993 \\
Boat & 0.9973 & 0.9956 \\
Elaine & 0.9984 & 0.9954 \\
House & 0.9980 & 0.9943 \\
Jet & 0.9965 & 0.9964 \\
Peppers & 0.9980 & 0.9947 \\
Average & 0.9979 & 0.9960 \\
\hline
\end{tabular}

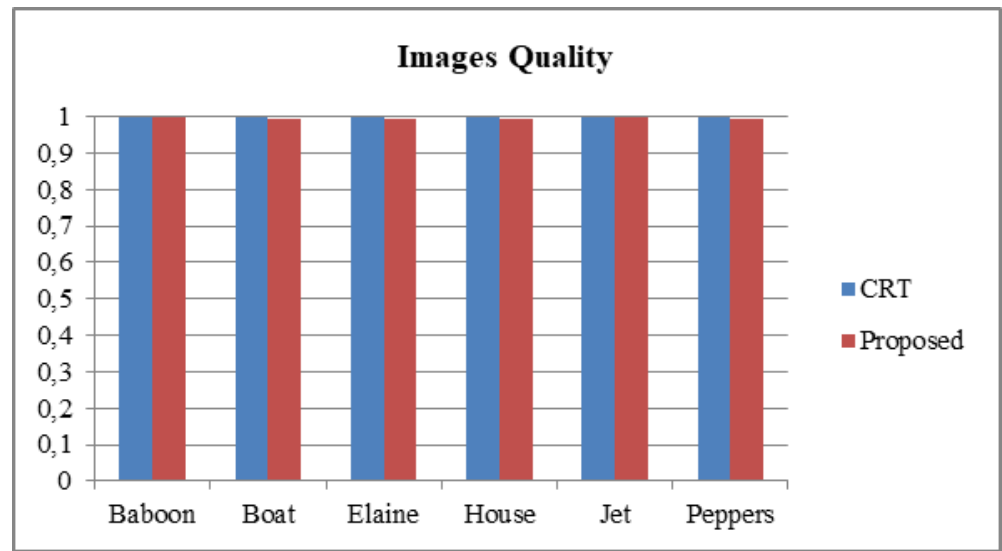

Figure 6. Images quality

The location of the most significant coefficients are the mapped into LL to locate the coefficients that will be embedded. The selected coefficients are the highest frequency of down sampled pixel. They are able to reduce the degradation of structural similarity which is in turn maintains the imperceptibility.

\section{CONCLUSION}

This paper is proposed a new watermarking scheme to embed the watermark through dual band IWT. The watermark is embedded on the low frequency sub band which is indexed using the most significant coefficients of high frequency sub band. In robustness test, the CRT method result has the average NC value of $0.7129,0.4846$, and 0.6768 while the proposed method has higher NC value of $0.7902,0.7473$, and 0.8163 in corresponding Gaussian filter, JPEG, and JPEG2000 compression test. Meanwhile the both CRT and proposed method have similar average SSIM value of 0.9979 and 0.9960 respectively in term of image quality. The result shows that the proposed method has higher robustness and able to maintain the image quality.

\section{ACKNOWLEDGMENT}

The authors would like to thanks to Ministry of Research, Technology, and Higher Education (RISTEKDIKTI) of Indonesia for funding this study through a Research Grants Scheme (SP DIPA042.06.1.401516/2017).

\section{REFERENCES}

[1] A. Yudhana, Sunardi, and S. Saifullah, "Segmentation Comparing Eggs Watermarking Image and Original Image," Bulltein Electr. Eng. Informatics (BEEI), vol. 6, no. 1, pp. 47-53, 2017.

[2] V. Singh, R. Kumar, and A. Ojha, "Significant region based robust watermarking scheme in lifting wavelet transform domain,” Expert Syst. Appl., vol. 42, no. 21, pp. 8184-8197, 2015. 
[3] S. D. Lin, "A robust DCT-based watermarking for copyright protection," IEEE Trans. Consum. Electron., vol. 46, no. 3, pp. 415-421, 2000.

[4] J. C. Patra, J. E. Phua, and C. Bornand, "A novel DCT domain CRT-based watermarking scheme for image authentication surviving JPEG compression,” Digit. Signal Process., vol. 20, no. 6, pp. 1597-1611, 2010.

[5] L. Umaroh, Y. P. Astuti, C. A. Sari, and E. H. Rachmawanto, "A Robust Image Watermarking Using Hybrid DCT and SLT," in 2016 International Seminar on Application for Technology of Information and Communication (ISemantic), 2016, pp. 312-316.

[6] C.-C. Lai and C.-C. Tsai, "Digital Image Watermarking Using Discrete Wavelet Transform and Singular Value Decomposition,” IEEE Trans. Instrum. Meas., vol. 59, no. 11, pp. 3060-3063, 2010.

[7] M. Ali, C. W. Ahn, M. Pant, and P. Siarry, "An image watermarking scheme in wavelet domain with optimized compensation of singular value decomposition via artificial bee colony," Inf. Sci. (Ny)., vol. 301, pp. 44-60, 2015.

[8] C. Li, Z. Zhang, Y. Wang, B. Ma, and D. Huang, "Dither modulation of significant amplitude difference for wavelet based robust watermarking," Neurocomputing, vol. 166, pp. 404-415, 2015.

[9] A. O. Mulani and P. B. Mane, "Watermarking and Cryptography Based Image Authentication on Reconfigurable Platform,” Bulltein Electr. Eng. Informatics, vol. 6, no. 2, pp. 181-187, 2017.

[10] N. M. Makbol and B. E. Khoo, "A new robust and secure digital image watermarking scheme based on the integer wavelet transform and singular value decomposition," Digit. Signal Process., vol. 1, no. 134, pp. 1-14, 2014.

[11] M. Botta, D. Cavagnino, and V. Pomponiu, "A modular framework for color image watermarking," Signal Processing, vol. 119, pp. 102-114, 2016.

[12] N. Raftari and A. M. E. Moghadam, "Digital image steganography based on Integer Wavelet Transform and assignment algorithm," Proc. - 6th Asia Int. Conf. Math. Model. Comput. Simulation, AMS 2012, pp. 87-92, 2012.

[13] Z. Wang, A. C. Bovik, H. R. Sheikh, and E. P. Simoncelli, "Image Image quality assessment: From error visibility to structural similarity,” IEEE Trans. Image Process., vol. 13, no. 4, pp. 600-612, 2004.

[14] J. Xu, A. H. Sung, P. Shi, and Q. Liu, "JPEG compression immune steganography using wavelet transform," Int. Conf. Inf. Technol. Coding Comput. 2004. Proceedings. ITCC 2004., vol. 2, 2004.

[15] N. A. Abu, P. W. Adi, and O. Mohd, "Robust Digital Image Steganography within Coefficient Difference on Integer Haar Wavelet Transform," International J. Video Image Process. Netw. Secur., vol. 14, no. 2, pp. 1-8, 2014.

[16] P. W. Adi, F. Z. Rahmanti, and N. A. Abu, "High Quality Image Steganography on Integer Haar Wavelet Transform using Modulus Function," in 2015 International Conference on Science in Information Technology (ICSITech), 2015, pp. 79-84.

[17] P. W. Adi and F. Z. Rahmanti, "Robust Integer Haar Wavelet Based Watermarking using Singular Value Decomposition,” J. Ilmu Komput. dan Inf., vol. 9, no. 1, pp. 26-34, 2016.

[18] S. G. R. Ekodeck and R. Ndoundam, "PDF steganography based on Chinese Remainder Theorem," J. Inf. Secur. Appl., vol. 29, pp. 1-15, 2016.

[19] X. Li, W. Wang, W. Wang, X. Ding, and Q. Yin, "Optimal estimates of common remainder for the robust Chinese Remainder Theorem,” Commun. Nonlinear Sci. Numer. Simul., vol. 19, no. 7, pp. 2373-2381, 2014.

[20] J. C. Patra, A. Karthik, and C. Bornand, "A novel CRT-based watermarking technique for authentication of multimedia contents,” Digit. Signal Process., vol. 20, no. 2, pp. 442-453, 2010. 